\title{
Technological Innovations in Metals Engineering
}

\author{
BRETT CONNER (1) ${ }^{1,13}$ SHAI ESSEL, 2 SHILADITYA PAUL ${ }^{2},{ }^{3,4}$ \\ RAFAEL WERTHEIM ${ }^{5}$ TIM PASANG ${ }^{6}$ DENNIS P. BUTCHER JR. ${ }^{7}$ \\ HENNING ZEIDLER, ${ }^{8}$ NARENDRA DAHOTRE, ${ }^{9}$ SARANG PANDE, ${ }^{10}$ \\ RAJARSHI BANERJEE ${ }^{9}$ SANTIAGO CUESTA-LOPEZ, ${ }^{11}$ \\ NORBERT BABCSAN, ${ }^{12}$ and VIRGIL SOLOMON ${ }^{1}$
}

\begin{abstract}
1.-Advanced Manufacturing Research Center, Youngstown State University, Youngstown, $\mathrm{OH}$ 44555, USA. 2.-Technion Israel Institute of Metals, Technion City, 32000 Haifa, Israel. 3.-The Welding Institute (TWI), Granta Park, Great Abington, Cambridge CB21 6AL, UK 4.-The University of Leicester, University Road, Leicester LE1 7RH, UK. 5.-Fraunhofer IWU, Reichenhainer Str. 88, 09126 Chemnitz, Germany. 6.-Engineering Research Institute, Auckland University of Technology, 55 Wellesley Street East, Auckland Central, New Zealand. 7.-Air Force Research Laboratory, Materials and Manufacturing Directorate, Wright-Patterson, Air Force Base, Dayton, OH 45433, USA. 8.-Technische Universität Bergakademie Freiberg, Agricolastrasse 1, 09599 Freiberg, Germany. 9.-Department of Materials Science and Engineering, University of North Texas, 1155 Union Circle \#305310, Denton, TX 76203-5017, USA. 10.-Marwadi Education Foundation, Marwadi University, Gauridad, Rajkot, Gujarat 360 003, India. 11.-ICAMCyL International Center for Advanced Materials and Raw Materials of Castilla y Leon, 24006 Leon, Spain. 12.-University of Miskolc, Miskolc 3515, Hungary. 13.-e-mail: bpconner@ysu.edu
\end{abstract}

\section{INTRODUCTION}

The quickening pace of technology advancement in metals is driven by digital processes and raw material supply pressures. Innovations in metals are being driven by forces such as materials informatics for alloy development, requirements for lightweight materials, and rapid manufacturing in the form of additive manufacturing (AM). At the same time, innovations are also being driven by global trends. For example, supply of critical raw materials forces alloy development or alternative materials, especially where these materials are needed for extreme environments or extreme performance. The metals industry needs to be aware of innovations and how they can enhance or disrupt their business.

Production of functional metal parts by AM provides a range of benefits including increased functionality and light weighting through geometric complexity, production quantity flexibility and affordable low-volume production, customization, point-of-need fabrication, and on-demand production. ${ }^{1,2}$ As with any new process, there is a need to understand the relationships between process, structure, properties, performance, and cost or economical aspects, and how industry can take

Brett Conner is the guest editor for the invited topic Technological Innovations in Metals Engineering in this issue. advantage of those relationships to produce differentiated products. In aerospace, medicine, and energy, metal AM parts for critical applications must undergo qualification and certification. ${ }^{3,4}$ Research is needed to provide confidence in component design and in the material properties for process parameters that are known, monitored, and controlled. In addition, a few alloys have been developed to specifically take advantage of AM processes, and this is especially true for metal powders.

Millennia-old processes of castings, forgings, and sheet metal forming are being combined with advanced process simulation and additive manufactured tooling. Wireless sensors are being integrated into 3D-printed sand molds. ${ }^{5}$ In-envelope hybrid machine systems are being commercialized with subtractive machining production centers integrated with directed energy deposition of metals. Incremental sheet metal forming has been incorporated into a five-axis Lasertec 65 system laser powder deposition system which also includes subtractive milling and roller burnishing. ${ }^{6}$ Additively manufactured tubes are shown to be flow-formed, as described in this special edition.

Certain metals and metal alloying elements have a long- and even short-term supply which is at jeopardy. These critical raw materials (CRM) are typically key materials for industrial use. Threats to CRM supply can vary from health and 
environmental hazards and regulations, geo-political situations especially in areas producing the vast share of the CRM's global demand, scarcity of resources or costly extraction, and increase in demand. Innovations that address a CRM challenge can aim to change either supply or demand. Developments to improve the cost efficiency or environmental friendliness of CRM production or innovations in increased recyclability can solve supply threats. For key materials such as permanent magnets or cemented carbides, there are research challenges to reduce CRM demand by replacement of these elements or reduction of the needed amount. In most production cases, the transition from classic manufacturing to additive is known to reduce CRM consumption as the buy-tofly ratio is dramatically lower using AM.

Maximizing the service life of metal components remains a critical challenge. Corrosion remains a concern, and corrosion mitigation is essential as corrosion costs roughly $3.4 \%$ of the global GDP and, more importantly, presents a threat to the environment and public safety. ${ }^{7}$ Corrosion mitigation by cost-effective production of metallic coatings has been the focus of several materials and corrosion research projects in recent years. Metallic coatings for corrosion mitigation are commonly deposited by electroplating, electroless plating, spraying, hot dipping, and physical and chemical vapor deposition. Although some of these techniques are mature, new developments in coating production are ongoing. The techniques for production of metallic coatings are numerous, and each one has its advantages and drawbacks. The selection of the coating technique is critical and is often made on the basis of cost while still meeting service performance requirements. Prudent selection of coating materials and deposition processes can lead to improved performance and long service life while reducing the overall life-cycle cost.

In an environment of rapid change, it is vital for the metals industry to identify technical gaps, to gain awareness of technologies that could enhance their products and processes or even disrupt the industry, and to create avenues of technology adoption. Innovation sources such as universities, national laboratories, non-profit research institutions, small businesses, and industrial laboratories can hold the keys to solve industrial challenges and provide competitive advantage. In many conference forums, relatively high technology readiness level or manufacturing readiness level innovations are not sought out and therefore the technology transition "Valley of Death" is reinforced.

The Technion Israel Institute of Metals and the Youngstown State University, in collaboration with TMS, established the Technical Innovations in Metals Engineering (TIME) conference to identify both the needs of industry as well as the state of the art. To deepen the CRM discussion, TIME 2018 was organized with partnership of CRM-Extreme, EC
COST action 15102, which brings together European experts in the CRM fields relevant to extreme working conditions and coordinated by the Università Politecnica delle Marche from Ancona, Italy. TIME 2018 had four focus areas:

- Additive manufacturing.

- Metals processing through shaping, forming, and solidification.

- Alloys and critical materials.

- Applications.

This first-ever conference was held in Haifa, Israel, in May 2018 and nearly 250 individuals attended.

The articles contained in this special topic are invited papers from this conference. TIME 2020 will be held in Youngstown, Ohio.

\section{ARTICLES INCLUDED UNDER THE TOPIC OF TECHNOLOGICAL INNOVATIONS IN METALS ENGINEERING}

The topic of laser powder bed fusion additive manufacturing for the purpose of medical implants is addressed in "Towards Qualification of Additively Manufactured Ti6Al4V (ELI) Medical Implants" by W. Du Preez. An overview of qualification protocol research in South Africa is given in this paper. For example, the researchers addressed the quality management of powders using ISO 13485 and examined how particle size distribution and porosity evolved over the course of multiple reuse cycles. This research contributed to the qualification and approval for the first maxillofacial AM implant in South Africa.

Another medical approach is taken in " $3 \mathrm{D}$ Bioprinting of Hybrid Materials for Regenerative Medicine. Implementation in Innovative SMEs" by R.M. Piticescu et al. Here, hydroxyapatite and polyurethane-diol are synthesized and then printed using a syringe-based material extrusion (i.e., bioplotting) technology. Ocular implants are a targeted application of this technology.

High-entropy alloys (HEAs) are being explored due to their high-temperature strength and creep resistance. Newer generations of HEAs are discussed in "Effect of $\mathrm{Zr}$ on the Microstructure of Second and Third Generation BCC HEAs" by E. Eyal et al. These newer alloys have been developed to reduce density and increase corrosion behavior. Density is lowered by eliminating or reducing the content of W, Mo, and Ta. Corrosion behavior can be enhanced by Zr. Here, researchers look at the evolution of microstructure evolution when HEAs are heated to $1000^{\circ} \mathrm{C}$ or $1300^{\circ} \mathrm{C}$ and then quenched.

The extreme conditions found in the high-temperature applications of transportation, energy, and manufacturing operations require thermal protective systems for metallic structures. Ultra-hightemperature ceramics (oxide or non-oxide) are generally the preferred material choice. "Strategies for Development of Novel Material Systems and 
Coatings for Extreme Environments" by R. R. Piticescu et al. examines the selection of ultrahigh-temperature ceramics and processing approaches. The researchers then present original research on the processing of ultra-high-temperature ceramics based on barium and lanthanum zirconates with perovskite structure through the use of electron beam vapor deposition.

"Solidification During Selective Laser Melting of Co-29Cr-6Mo alloy" by Z. Chen et al. focuses on laser powder bed fusion of an alloy and the process combination being used in medical and aerospace applications. This research examines how process parameters influence grain size and directional growth. Maps of solidification front thermal rates and gradients are presented.

Titanium welding has been leveraged by industry for aerospace, chemical industrial, and marine or offshore applications. For $\alpha$ alloys, near- $\alpha$ alloys and $\alpha+\beta$ alloys, the fusion zone and heat-affected zone show high hardness compared with the base metal. $\beta$ alloys, however, have fusion zones and heataffected zones with lower hardness compared to base metal. For large-size components, post-welding heat treatment to create a more uniform hardness across the weldment is not practical. In "Improvements in Welding Properties of Titanium Through the Use of Interlayers" by T. Pasang et al., it is shown that, by selecting a proper titanium alloy interlayer, the above issues can be minimized, and the need for post-welding heat treatment may be reduced or eliminated, which will lead to cost reduction and process efficiency.

Wire and Arc Additive Manufacturing has potential for the printing of large-scale parts because of its relatively high metal deposition rates and low costs. In the paper "Influence of Shielding Gas Composition on Microstructure and Mechanical Properties of Wire and Arc Additive Manufactured Inconel 625”, I. Jurić et al. explore the geometric and mechanical performances of Inconel 625 built up using four different shielding gas combinations with a MIG/MAG process.

The potential of hybrid approaches involving additive and conventional forming can be shown in "From Wire to Seamless Flow-Formed Tube: Leveraging the Combination of Wire Arc Additive Manufacturing and Metal Forming" by A. Shirizly and O. Dolev. Wire-arc AM was used to produce from steels two types of pre-forms: a closed tube and an open cylinder. These were then flow-formed and characterized.

Finally, roll bonding of aluminum sheets is used by industry to create corrosion-resistant aerospace sheets, high-strength or crash-resistant automotive sheets, and heat exchangers. The mechanical performance of these sheets is strongly dependent on the interface bonding strength between them. "A Computational Analysis of Thermo-Mechanical Fields in Hot Roll Bonding of Aluminum Validated by Experiments" by B. Mittelman et al. presents coupled thermo-mechanical computational modeling of a hot roll bonding process of aluminum sheets validated by experiments.

The following papers are published under the topic "Technological Innovations in Metals Engineering" in the February 2019 issue (vol. 71, no. 2) of $J O M$ and can be accessed via the $J O M$ page at http://link.springer.com/journal/11837/71/2/page/1.

- "Towards Qualification of Additively Manufactured Ti6Al4 V (ELI) Medical Implants" by Wilhelm Bouwer du Preez

- "3D Bioprinting of Hybrid Materials for Regenerative Medicine: Implementation in Innovative Small and Medium-Sized Enterprises (SMEs)" by Roxana Mioara Piticescu, Laura Madalina Cursaru, Dan Nastase Ciobota, Sinziana Istrate, and Dumitru Ulieru

- "Effect of Zr on the Microstructure of Second and Third Generation BCC HEAs" by Eyal Eshed, Natalya Larianovski, Aleksey Kovalevsky, and Alexander Katz Demyanetz

- "Strategies for Development of Novel Material Systems and Coatings for Extreme Environments" by Radu R. Piticescu, Marina Urbina, Antonio Rinaldi, Santiago Cuesta-Lopez, and Arcadii Sobetkii

- "Solidification During Selective Laser Melting of Co-29Cr-6Mo Alloy" by Zhan Chen, Teresa Guraya, K. Darvish, M. Phan, and T. Pasang

- "Improvements in Welding Properties of Titanium Through the Use of Interlayers" by Timotius Pasang, Mona Aziziderouei, Yuan Tao, Dominique Ogletree, Gorja Stump, Yuji Sato, Masahiro Tsukamoto, and Cho-Pei Jiang

- "Influence of Shielding Gas Composition on Microstructure and Mechanical Properties of Wire and Arc Additive Manufactured Inconel 625" by Ivan Juri, Ivica Garašić, Matija Bušić, and Zoran Kožuh

- "From Wire to Seamless Flow-Formed Tube: Leveraging the Combination of Wire Arc Additive Manufacturing and Metal Forming" by A. Shirizly and O. Dolev

- "A Computational Analysis of Thermo-Mechanical Fields in Hot Roll Bonding of Aluminum Validated by Experiments" by Brigit Mittelman, Gilad M. Guttmann, and Elad Priel

\section{REFERENCES}

1. I. Gibson, D. Rosen, and B. Stucker, Additive Manufacturing Technologies: 3D Printing, Rapid Prototyping, and Direct Digital Manufacturing [Internet]. Springer; 2014. https://ma rket.android.com/details?id=book-OPGbBQAAQBAJ.

2. B.P. Conner, G.P. Manogharan, A.N. Martof, L.M. Rodomsky, C.M. Rodomsky, D.C. Jordan, and J.W. Limperos, Additi. Manuf. 1-4, 64-76 (2014). https://doi.org/10.1016/j.add ma.2014.08.005.

3. M. Seifi, A. Salem, J. Beuth, O. Harrysson, and J.J. Lewandowski, JOM 68, 747-764 (2016). https://doi.org/10.1007/ s11837-015-1810-0.

4. W.E. Frazier, J. Mater. Eng. Perform. 23, 1917-1928 (2014). https://doi.org/10.1007/s11665-014-0958-z. 
5. J. Walker, E. Harris, C. Lynagh, A. Beck, R. Lonardo, B. Vuksanovich, J. Thiel, K. Rogers, B. Conner, and E. MacDonald, Int. J. Metalcast. 12, 785-796 (2018). https://doi. org/10.1007/s40962-018-0211-x.

6. D.Y. Yang, M. Bambach, J. Cao, J.R. Duflou, P. Groche, T. Kuboki, A. Sterzing, A.E. Tekkaya, and C.W. Lee, CIRP Ann. 67, 743-765 (2018). https://doi.org/10.1016/j.cirp.2018.05.004.
7. G. Koch, J. Varney, N. Thompson, O. Moghissi, M. Gould, and J. Payer, International Measures of Prevention, Application, and Economics of Corrosion Technologies Study, IMPACT Report (Houston, TX: NACE International, 2016). 